\title{
Some Thoughts on the Application of Three-dimensional Technology in the Construction of Transmission and Transformation Engineering
}

\author{
Zhang Wuchen, ${ }^{1}$ Wu Gaofeng, ${ }^{2}$ Wang Gang, ${ }^{3}$ Xing Lin, ${ }^{1}$ Li Mingfu ${ }^{1}$ \\ ${ }^{1}$ State Grid Hebei Economics Research Institute, 050000 Shijiazhuang, Hebei, China \\ ${ }^{2}$ State Grid Hebei Electric Power Co., Ltd, 050000 Shijiazhuang, Hebei, China \\ ${ }^{3}$ State Grid Hebei Construction Company, 050000 Shijiazhuang, Hebei, China
}

\begin{abstract}
This paper puts forward some thoughts on the fine management of power transmission and transformation engineering construction with the help of three-dimensional technology, and discusses the installation, quality acceptance and construction organization deepening application of steel components in power transmission and transformation engineering construction. The physical 3D model is established by scanning technology, and the accuracy of machining components is checked by comparing the design model with the physical model. The construction process is simulated by the three-dimensional engineering management platform, and the problems existing in the actual construction are found, and the optimization of the construction organization design is carried out through the platform.
\end{abstract}

\section{Introduction}

With the increasing demand for electricity, the scale of power grid construction is gradually expanding. In order to build a strong smart grid, the technology, progress, quality and safety of engineering construction are constantly put forward higher requirements. The times are progressing, and the control methods of infrastructure projects are also developing. In recent years, engineering three-dimensional technology has developed rapidly, and the demand for this technology has become increasingly urgent and has a good application prospect. However, the application of 3D technology in power grid engineering construction industry is still very limited, mainly concentrated in the stage of engineering design, and needs to be deepened and popularized in continuous exploration. ${ }^{[1]}$

\section{Application of 3D Technology in Power Grid Construction}

In the era of big data, engineering construction needs to use advanced digital technology to gradually improve the quality of construction and management, to consider the whole life cycle of the project as a whole, and to create greater value for infrastructure projects through information model and other technologies.

Recently, the electric power survey and design industry has explored this, from BIM (Building Information Modeling) to EIM (Engineering Information Modeling), the whole process of power grid engineering construction has been studied by three-dimensional digitization, but it has not been applied in practical engineering on a large scale.

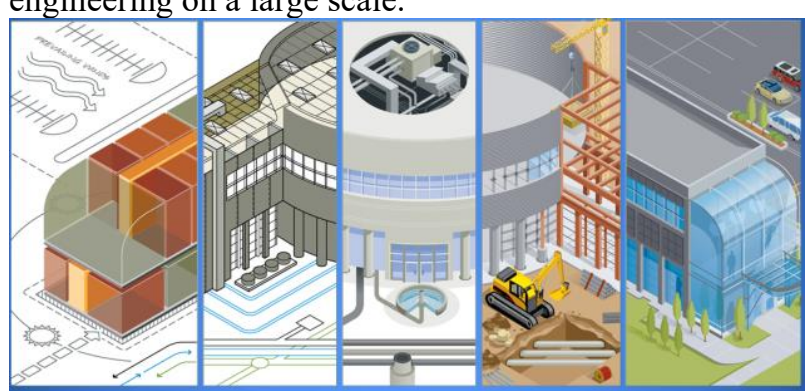

Fig. 1 Application of 3D technology in engineering construction

BIM improved the vertical and horizontal communication and multi-dimensional communication between the management system and workflow in the whole project from the early planning to the final operation and maintenance stage, ensured the normal operation of each link in each stage of the whole life cycle of the project, and realized the information management of the whole life cycle of the project. ${ }^{[2]}$ Based on the three-dimensional digital technology, the BIM digitally expresses the building products and their functional characteristics in order to realize the information sharing between all stages of the whole life of the building and the participants. The 4D model of construction engineering based on 4D-CAD technology is one of the most important supporting technologies BIM applied in the construction stage. ${ }^{[3-4]}$

A lot of application software based on BIM concept has been developed, which has gradually become perfect

* Corresponding author: Zhang Wuchen,499797324@qq.com 
in structural analysis, collision inspection, engineering quantity statistics, construction simulation, visual bottom and so on.Although the current mainstream 3D design software can realize most of the functions of substation design, there are still some problems such as low efficiency, insufficient intelligence and less interface with technical and economic professional software. The software function needs to be further expanded, especially in the whole industry chain and the intelligent software function. ${ }^{[5]}$

In addition, the application of construction, operation and maintenance is still in the exploration stage. The application of BIM technology in construction site planning, construction process simulation and construction cooperative management will be a key point in the future development.

\section{Use 3D technology to improve the accuracy of material processing}

The steel structures such as high and low voltage side outlet structure, lightning rod and line tower in substation are usually preassembled in two-dimensional mode in the factory area after the factory processing is completed, In order to test the accuracy of material processing. However, this method has an obvious defect, for the three-dimensional steel structure frame, it is easy to find in the assembly process that the steel structure angle error can not be used, affecting the construction progress, resulting in the site nest, construction cycle extension of the events occur from time to time.Moreover, the pre-assembly of steel components takes more time, and the corresponding personnel and lifting equipment are needed to complete the work, which costs a lot.

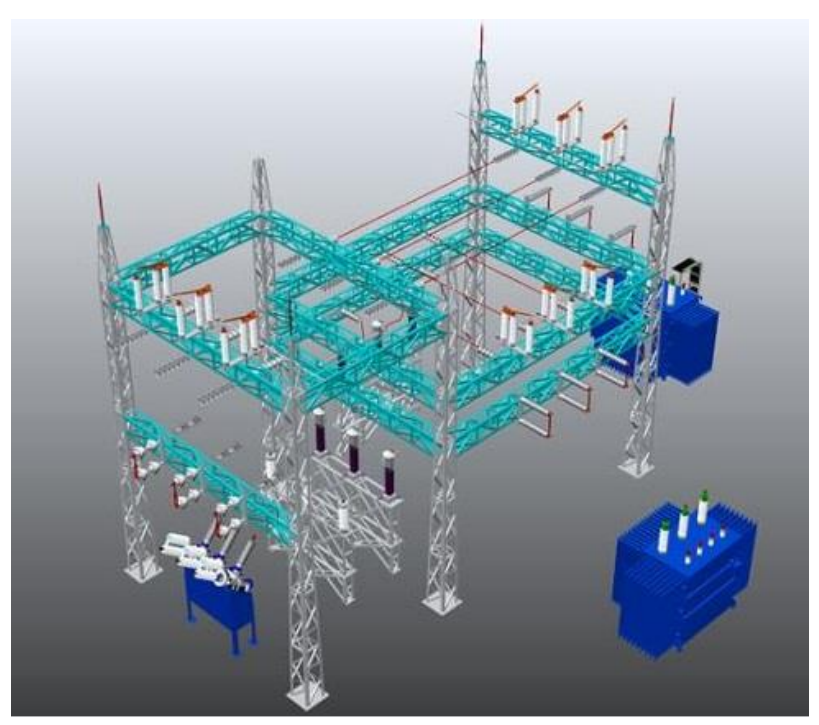

Fig. 2 3D preassembly model of the structure

In view of this problem, we can use $3 \mathrm{D}$ measurement technology to realize virtual assembly in data platform.Combined with the design model established in the design stage, the processing manufacturer will deepen the design of the model before the actual production and processing, and form a deepening processing model to meet the requirements of processing and production. After processing the steel according to the processing model, the factory can scan, number and input all kinds of attribute parameters through the machine, and establish the physical model library of all the components. The components in the physical model library are simulated and assembled on the data platform according to the design model to check the qualification of the processed components and to simulate some problems in the process of construction assembly. Thus, we can know whether to adjust the processed components or whether to further optimize the construction organization.

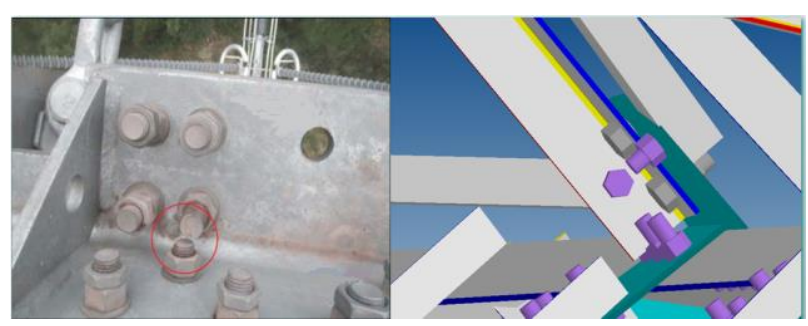

Fig. 3 Structural preassembly simulation

This not only saves the time of pre-assembly of tower, shortens the period from tower material processing to transportation to construction site, but also saves the manpower invested in checking the qualification of components. By inputting the material properties of each processing component, the deformation of each component of tower material in different stages can be simulated when the data platform is preassembled. Check whether the design model size and the actual machining component size can fully meet the requirements of field operation in the actual assembly process. Through these simulations, not only the accuracy of material processing is improved, but also the possible problems in the construction process can be clearly found, so as to facilitate the corresponding measures to be taken in advance in the construction process, so as to improve the fine level of construction management.

\section{Application of 3D Technology in Engineering Quality Acceptance}

The current acceptance of power transmission and transformation projects requires many different construction participants to carry out this work, involving multi-level acceptance. The acceptance process is complex and requires more manpower and time. The current construction acceptance work is only through the abstract two-dimensional model, and the acceptance data is scarce.

State Grid put forward new requirements to reduce the acceptance level and improve the efficiency of acceptance under the premise of ensuring the quality of work.Three-dimensional technology can help solve this problem. In the process of construction, the finished components of each construction structure are scanned 
by instruments, and the scanning data and images are input into the three-dimensional technical platform to form the three-dimensional model of the finished product. Then the model is compared with the construction drawing design model to verify whether the construction meets the requirements of the design model.

At the same time, the shape and surface condition of the physical model can be identified by intelligent software technology, such as the honeycomb hemp surface condition, the surface smoothness, the regularity of the shape and the accuracy of the size of the concrete foundation.

For the hidden project, after scanning the physical object before concealment, the physical 3D model is established to make it visible in the physical model of the whole project, and the construction parameters, material parameters, personnel information and so on are entered as the basis for the quality acceptance in the future.

After the physical 3D model is established by scanning, the acceptance of different levels of engineering only needs to be checked against the 3D model on the software platform. Through the input of the model time parameters, personnel information, material performance indicators, component coding, sampling test data and other information in the construction process, we can clearly see whether the quality of the construction process meets the requirements. After the acceptance of the three-dimensional model is completed, the acceptance of the whole project can be completed by on-site inspection or test according to the actual situation, thus greatly saving manpower and time cost. This is also in line with the State Grid corporation of the whole process of infrastructure integrated digital management concept.

\section{Simulation of construction organization design using 3D technology}

In the construction process of power transmission and transformation project, the construction site can be modeled by GIS through three-dimensional technology, and the characteristics and usage conditions of the site can be evaluated, so as to make the most ideal construction site planning and layout.

Using the three-dimensional design platform and combining the construction organization design to simulate the construction process, this paper analyzes what problems may occur in the whole process, such as the external space environment conditions, whether the site layout and the construction operation content are contradictory, the matching degree between the personnel arrangement and the construction schedule, and the route of the construction machinery entering and leaving the site. The construction unit can schedule the construction period according to the total workload and the working efficiency of different types of workers.

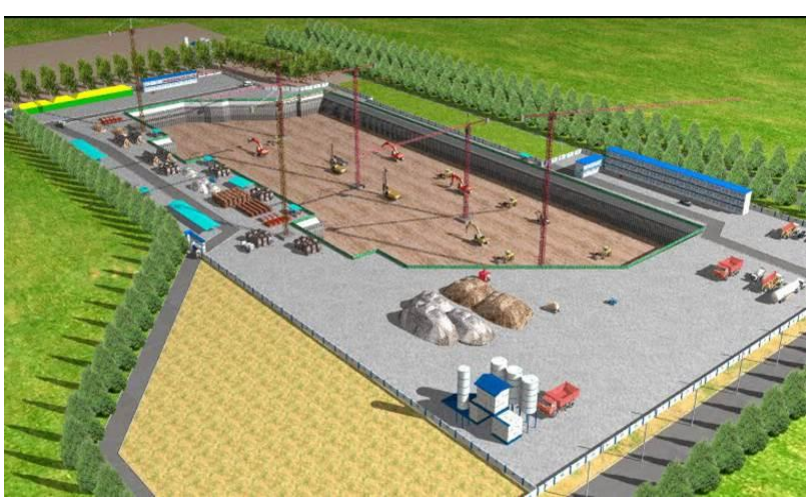

Fig. 4 3D construction layout simulation

The planning and layout of temporary facilities change dynamically with the progress of the site project. Sometimes it is necessary to retest the actual situation of the site in order to plan the site layout again. When there are special circumstances in the construction process, such as sometimes it is necessary for the crane to temporarily enter the field to carry out some hoisting operations, but there is a lack of organizational measures for such temporary events in the whole construction organization design. This requires temporary adjustment of site organization. At this time, with the help of the three-dimensional simulation platform, the simulation can be carried out in the three-dimensional model. Through the software analysis, whether the entry of the crane will affect the operation of other adjacent processes, and if so, how to solve it.

At the same time, a specific construction node in the construction process can be simulated, especially the difficulties in construction, to achieve the overall control of local operations.For example, scaffolding engineering, when using 3D management platform for construction organization simulation, we can input the characteristic information, design code basis, material physical and mechanical characteristics, construction scheme and so on. These parameters can be used as the basis of simulation calculation, and the safety and stability of the support system can be analyzed by mechanical calculation and simulation. Then the software automatically calculates the amount of scaffolding materials, including the number of steel pipes, the number of fasteners, which is conducive to the unified management of the materials in the construction site, and at the same time, according to the simulation analysis, reasonable arrangement of labor, to achieve the fine management of the local project.

\section{$63 \mathrm{D}$ analysis based on FEM is used to simulate foundation pit support}

Substation sites are usually limited by the overall planning of the government, so it is difficult to ensure that all sites are flat, even soil and have good foundations. There are often a lot of bad geological conditions in many sites, if not handled or not handled properly, it will bring a lot of potential risks to substation construction and post-operation. In the process of substation construction, in case of coastal and river 
alluvial plain, pile foundation is often used for electrical equipment to control foundation deformation, and the ground is consolidated. In the process of construction, some deep foundation pits need to be supported to ensure the safety of personnel and structure. Compared with the site with good site conditions, the civil construction investment will be significantly increased. Therefore, it is particularly important to select an economically and technically reasonable effective foundation plan and conduct quantitative analysis and risk assessment of the deformation and stress of the structure in the station in view of the geologically poor areas. Three-dimensional numerical analysis technology can visually won't be able to directly identify the underground part, through the survey data analysis, more intuitively show the designer, convenient for designers to design, as well as construction personnel to carry out the corresponding construction provide intuitive technical guidance, understand points that should be paid attention to in the construction safety risk, so as to take corresponding measures in time, ensure the safety of the construction process.

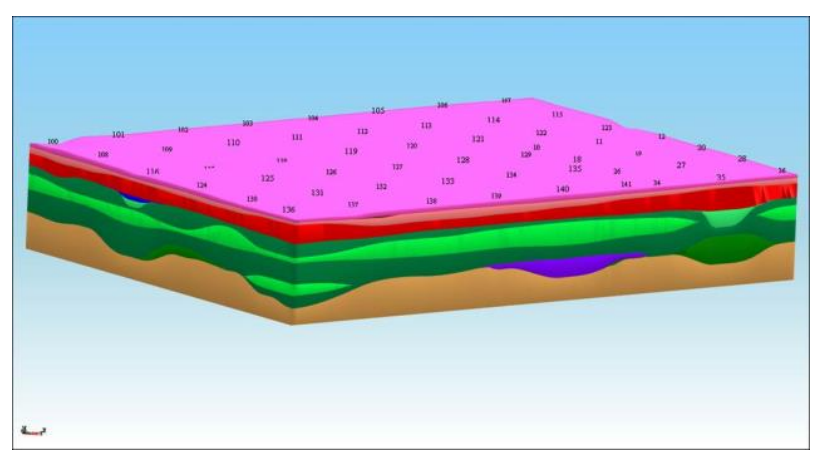

Fig. 5 3D geological model

By means of refined numerical modeling and simulation analysis of the whole construction process, not only can three-dimensional geological model and structural model be shown more intuitively than the traditional calculation book based on two-dimensional section design, but also the deformation and stress of the ground and structure can be transmitted from multiple angles and in various forms with the change of the whole construction process. With high precision in calculation, numerical simulation of stress and strain of soil constitutive model to describe the features, and can be easily considering soil and structure interaction, threedimensional effect, all kinds of external load and the influence of the groundwater pressure field, can overcome many simplifying assumptions do not tally with the actual situation in the traditional method, the calculation error of more realistic analysis results.

Natural foundation of substation engineering and deep foundation, foundation treatment, retaining method of numerical simulation technology research, can be more scientifically guiding foundation design and construction scheme selection and optimization, to improve the level of substation foundation design, improve the rationality and validity of the design scheme, to ensure normal operation of transformer substation and power supply safety and stability, has the important value of engineering application and social and economic benefits.

\section{Conclusion}

To sum up, continuous exploration and deepening of the application of three-dimensional technology can solve some problems in management and technology in current engineering construction, realize digital, information and visual management of power transmission and transformation engineering construction, make construction management more convenient, fast and accurate, and improve the technical level of engineering construction.It is of great significance to popularize and deepen 3D technology in power transmission and transformation engineering, which can solve some problems in management and technology in engineering construction at present, and improve the level of engineering construction. The application prospect is very broad.

\section{References}

1. He Ju, Qin Yang. Study on the Application of BIM in the Construction Management of Electric Power Engineering [J].]1 Technology and Enterprise ,2015(1):63-65.

2. Xu Deguang. Application of BIM technology in substation construction. [D]. North China University of Water Conservancy and Hydropower ,2017:13-14.

3. EASTMAN C ,TEICHOLZ P ,SACKS R , et al.BIM handbook 2th edition [M]. New Jersey: John Wiley \&Sons ,2010: 2-29.

4. ZANEN P P A ,HA R TMANN T,AL-JIBOURI S $\mathrm{H} \mathrm{S}$, et al .Using 4D CAD to visualize the impacts of highway construction on the public [J]. Automation in Construction ,2013,32(1): 136-144.

5. GE Bin, Shao Songtao, Shi Min. Application of 3D Design in Substation Engineering [j].1 Applied Energy Technology ,2018,11:50-54. 